\title{
The Four Layers Elevator Control System Design Based on S7-200 PLC Xianjie Feng
}

\author{
Department of Architectural Engineering Dongguan University of \\ Technology, Dongguan, 523808, China \\ email:fengxianjie@126.com
}

\begin{abstract}
Keywords: S7-200; Elevator Control System; PLC Ladder Diagram
\end{abstract}
\begin{abstract}
This paper analyzes the use of PLC technology in the design of the elevator system. Using the programmable controller to control the elevator, with simple wiring, programming, intuitive, easy extension, etc. When the building floor increases, the hardware wiring on the floor only need to increase the corresponding input signal; the original wiring does not need to change, only in the process of writing software to increase the function of the corresponding floor, so to change the place is relatively small . Use ladder diagram program can be very intuitive see operation process. The simulation results show that the elevator in adaptability, accuracy and reliability, reach the design requirements, shows that this design scheme is feasible.
\end{abstract}

\section{Introduction}

At present the elevator control system there are three main ways: relay control system, the microcomputer control system, PLC control system[1]. Relay control system due to high failure rate, poor reliability, control methods are not flexible and large power consumption and other shortcomings, has gradually been eliminated. Although the microcomputer control system has the strong function in the intellectual control aspect, but which has perturbation resistance poor, system design complex. The PLC control system because of high reliability, convenient operation and maintenance, the advantages of strong anti-interference, has become the most currently used in the elevator control system

\section{The Overall Design of the Elevator Control System}

This design uses the PLC to carry on the operation and the control to four elevators, its process can be divided into the following several steps:

Familiar with the controlled object, to develop control programs.

To determine the design of the system I / O points.

Select the PLC model.

Select the input and output devices and assign the I / O address of the PLC

System debugging.

The preparation of relevant technical documents.

\section{PLC Analog Expansion Module EM233, EM235}

Both module resolution is 12bit, are not using optical isolation technology, are not embedded with the CPU's intelligent module. Intelligent module with embedded CPU can directly realize digital filtering, limiting, alarming and other functions of the signal inside the module, and even can directly transmit and process the temperature input signals of various temperature sensors. The module is a 4-channel analog input And 1 analog output of the hybrid module[2]. The system does the following configuration: CPU224, EM235, then the four-way into the channel address is AIW0, AIW2, AIW4, AIW6, 1 channel out of the channel address AQW0, as shown in Table 1. 
Table 1. Expansion module EM233 EM235 link

\begin{tabular}{|l|l|l|l|}
\hline 4 In / 4 Out (Module 0) & 4 Analog Input (Module 1) & 4 Out (Module 2) & 4 Analog Input 1 Analog Output \\
\hline I2.0 Q2.0 & AIW0 & Q3.0 & AIW8 AQW0 \\
I2.1 Q2.1 & AIW2 & Q3.1 & AIW10 (module 3) \\
I2.2 Q2.2 & AIW4 & Q3.2 & AIW12 \\
I2.3 Q2.3 & AIW6 & Q3.3 & AIW14 \\
\hline
\end{tabular}

Connect extension module to the right side of the CPU to increase and analog I/O point, form the chain of I/O. For the same type of input and output module, the module of the I/O address depends on the I/O types and the positions of the module in the I/O chain. Both module EM233, EM235 didn't adopt photoelectric isolation technology, are not intelligent module with embedded CPU. Using photoelectric isolation technology can improve the reliability of system and A/D, D/A conversion data stability.

S7-200 CN series PLC expansion module size is shown in Figure 1.
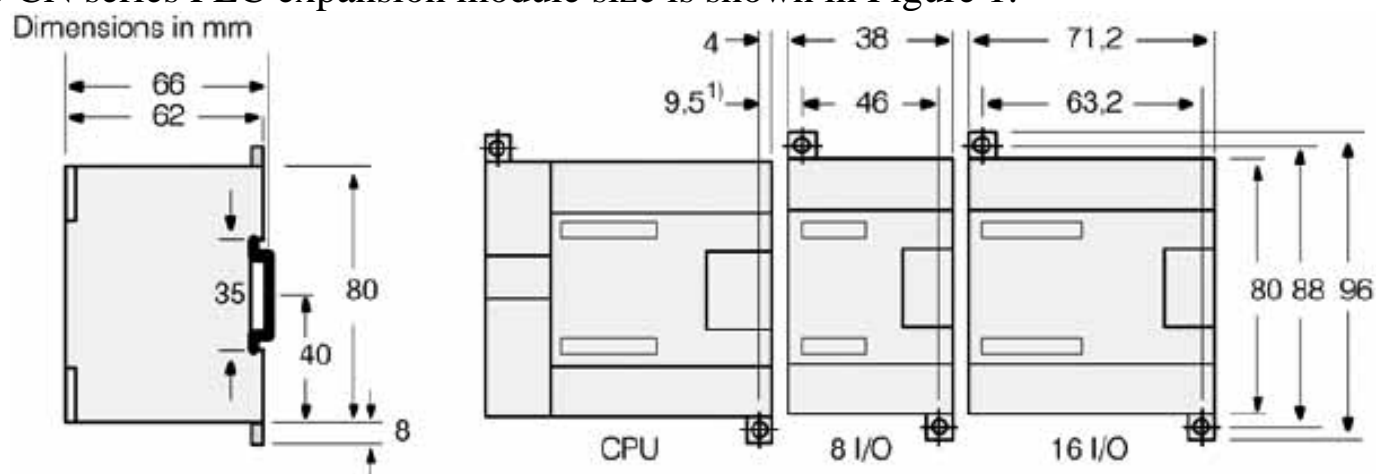

Fig.1. PLC expansion module size

\section{Hardware Configuration of Elevator Control System}

As shown in Figure 2, the hardware system is mainly composed of two parts, one part elevator control logic implemented by PLC[3]. Through the elevator linkage control program that enables the PLC to control the operation of the lift, and elevator speed control part through the high performance of vector control frequency converter, with pulse generator, so as to constitute a closed-loop vector control system of the motor, frequency control of motor speed operation (VVVF).

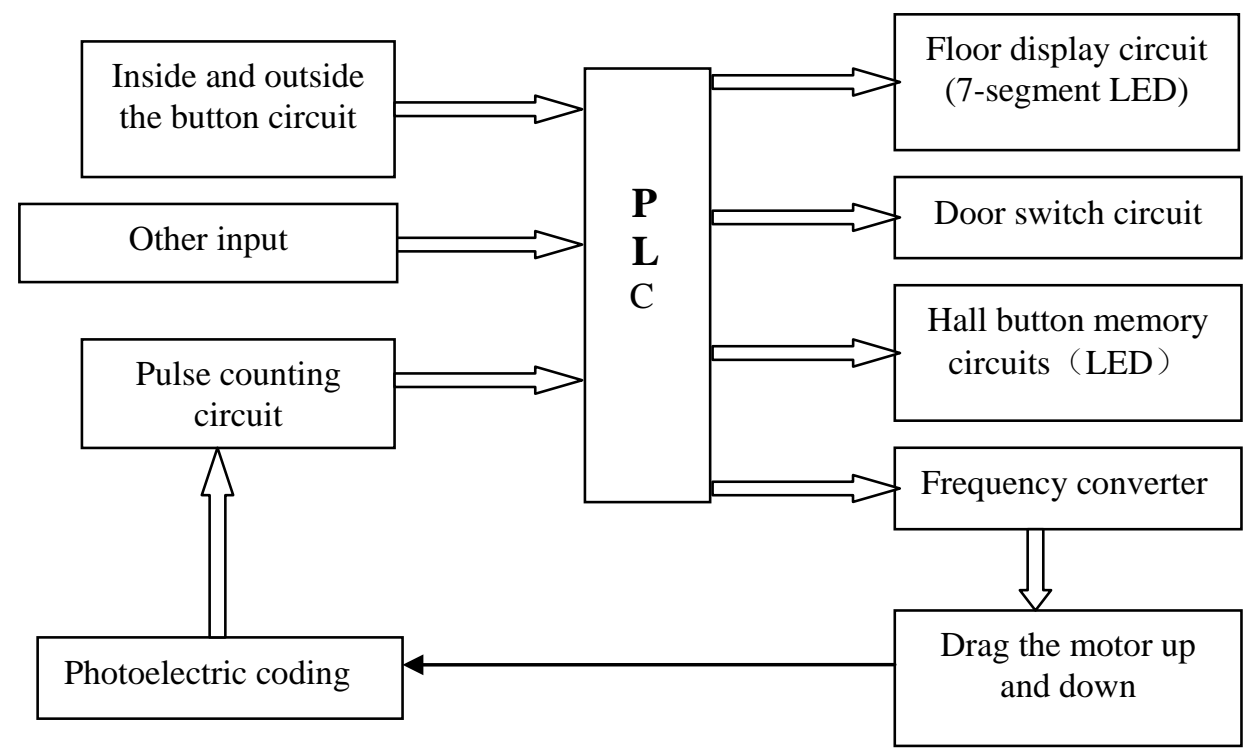

Fig.2. Hardware system 


\section{Elevator Control System Block Diagram}

The elevator system is mainly composed of the instructions in the circuit, the hall call circuit, main circuit, electrical machine button switch circuit, display circuit, memory, test and flat floor lamp circuit layer detection device and PLC circuit, etc, as shown in Figure 3.

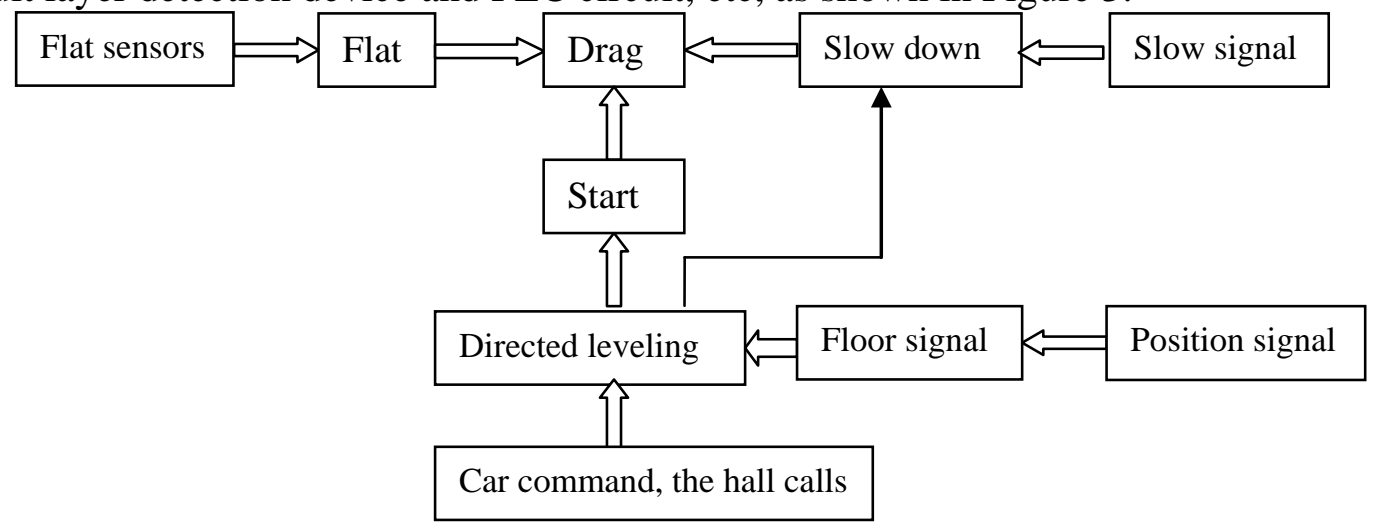

Fig.3. PLC circuit

\section{Calculation of PLC Control System I / O Points}

According to the characteristics of elevator control, the input and output signals are calculated as follows:

Inside and Outside the Car hall Call Button: The floor select button in the car has a total of 1 to 4 buttons, the layers of the hallway outside call button only rise in addition to the first floor button, the fourth floor only down button, the rest of the two layers are equipped with two buttons of rise and fall, therefore, a total of 11 need to input.

Position Signal: The position signal is generated by three sensors installed at the elevator parking position of each floor. Normally open, when the elevator to run flat to close. In addition there is a set of switch gate position, so the position signal requires a total of five inputs.

Elevator Door Control Signal: Most of the elevator has a door open button and close button, to facilitate manually open the door, so the elevator door control input signal must be a total of 2 .

Internal Call Indication Signal: There are four internal call signal, respectively, said 1 to 4 layers of the call is accepted, and the call command is completed, the signal disappears.

Outbound Call Indication Signal: There are 4 outbound call signals, respectively, that 1 to 4 layers of the outer call is accepted, and in the external call command is completed, the signal disappears.

Elevator Car Up and Down Control Signals: Elevator car up and down the instructions signal 2, the elevator up and down the line signal 2, door motor switch signal 2, a total of 6.

To sum up, a total of 32 input and output points.

\section{Safety Protection Devices for Elevators}

Electromagnetic Brake: mounted on the traction machine shaft, the general use of DC electromagnetic brakes, power-on when the release gate, stop power off after the brake[4].

Forced Deceleration Switch: installed on the top and bottom of the shaft respectively, when the car drove by end station in speed is not slow, capsules on collision block touches the switch, through electrical drive control device, motor forced to slow down.

Limit Switch: when capsules after end station smooth layer position still did not stop, the limit switch action, immediately cut off the power and braking, forced to stop.

Travel Limit Protection Switch: When the limit switch does not work, the car through the end station, the switch action.

Emergency Stop Button: Installed in the car driver control panel, abnormal circumstances, press the button to cut off the power supply, electromagnetic brake, elevator emergency stop. 
Hall Door Switch: Each hall door is equipped with a door lock switch. Only when the door closed to allow the elevator to start; in the event of the hall door switch off, the elevator immediately stop.

Close Door Safety Switch: Common is installed in the car door security panel, in the closing process, such as safety touch plate when the passenger, a signal, the door motor to close the door, reverse the door, delay re-opened the door, in addition to There are infrared switches and so on.

Overload Switch: when the overload when the car floor down switch action, the elevator can not close and run.

Other switches: safety window switch, belt pulley of the belt switch.

\section{The Inverter and the PLC Hardware Connection}

The inverter selection should pay attention to the following:

A. The purpose of using frequency, constant voltage control or constant current control.

B. The load type of the inverter, Pay attention to the performance curve of the load, and the performance curve will determine the way of application.

C. Inverter and load matching problems, voltage matching; current matching; torque matching.

Based on the above three points, and ensure no impact on the smooth start, no elevator reverse slip car, can slow down flat layer control, etc., the project selection is Panasonic VFO frequency converter series of frequency converter[5].

S7-200 integrated with high speed output function, and its output point Q0.0 and Q0.1 can form high speed pulse (the office) or pulse width modulation (PWM). The design of frequency converter and PLC hardware connection as shown in Figure 4.

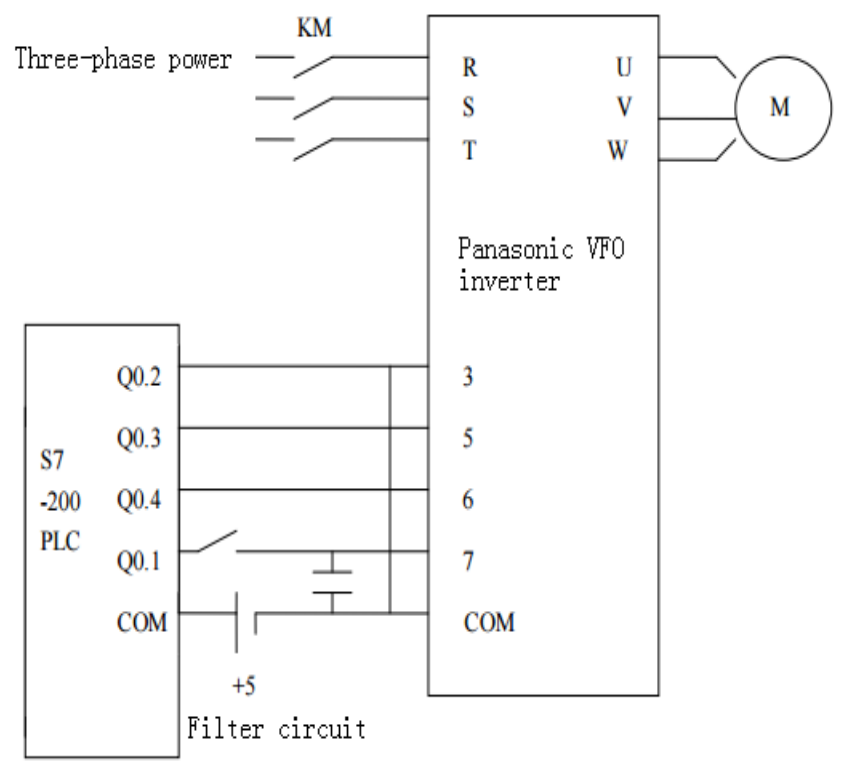

Fig.4. PLC hardware connection

\section{PLC Ladder Diagram of the Elevator Control}

The elevator floor position is controlled by travel switch. For example, if the elevator in the 2 flat layer (I1.5), a layer 2 indicator (Q3.1); When the elevator running upward (Q2.0), the light is still on, until the elevator to 3 layer flat (I2.0). This system PLC programming uses the ladder diagram language, the programming software is STEP 7. The software can complete the production process, the programmable controller CPU write / read, monitor program operation, debugger, PLC error diagnosis and a series of functions. The simulation result of the relevant ladder diagram is shown in Figure 5. 


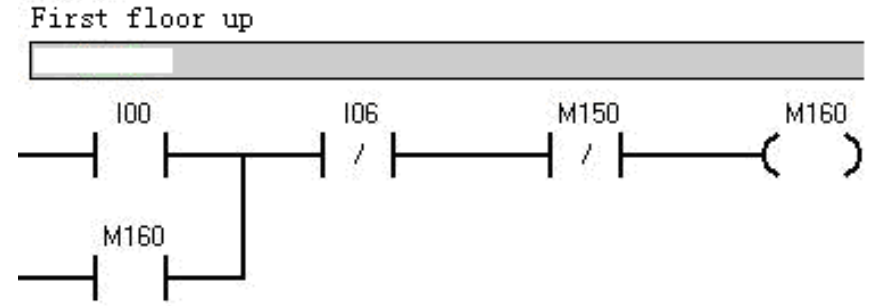

(a) First floor up

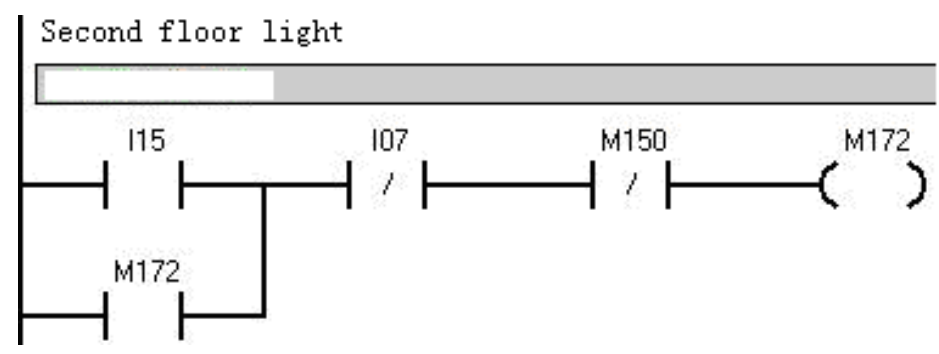

(c) Second floor light
Two floors down the request

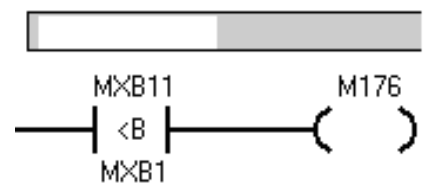

(b) Two floor down the request

Third floor down the request

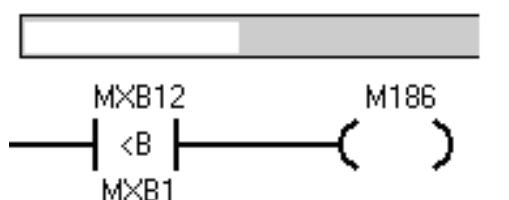

(d) Third floor down the request

Fig.5. PLC ladder diagram

\section{Conclusion}

The use of programmable logic controller (PLC) control technology transformation of the old elevator, make full use of modern power electronics technology, computer theory and detection technology to achieve a reliable control of the elevator.By reasonable equipment type selection, parameter setting and the software design, improve the operation reliability of the lift. After reforming PLC elevator compact structure, simple maintenance, low failure rate. If the PLC and ac frequency conversion speed regulation (VVVF) control technology will enhance the efficiency of the elevator and comfort, is advantageous to the energy saving of the lift.

\section{Acknowledgement}

This paper is aided by following Dongguan Institute of Technology "The key technology research of the mobile WiMAX system ", which project number: 2008108101006.

\section{References}

[1] Siemens AG.Working with STEP7 V5.2 Getting Started.2002.

[2] Matsushita Electric Works Ltd, Automation Control Group. FPO programming manual [EB/OL]. (2004-10-05)[2008-09-10].

[3] M. Paredes, M. Sartor, C. Masclet. An optimization process for extension spring design. Computer Methods in Applied Mechanics and Engineering. 2001, 191(8): 783-797.

[4] Joerg Christian Wolf, Phil Hall, Paul Robinson, Phil Culverhouse. Bioloid based Humanoid Soccer Robot Design, 2007.

[5] He yong yi. A Control System of Material Handling in FMS. Journal of shanghai university. Vol.1.No.1.1997. 